\title{
Research Article \\ Existence of Periodic Solutions for the Duffing Equation with Impulses
}

\author{
Xuxin Yang, ${ }^{1,2}$ Weibing Wang, ${ }^{3}$ and Jianhua Shen ${ }^{4}$ \\ ${ }^{1}$ Department of Mathematics, Hunan First Normal University, Hunan, \\ Changsha 410205, China \\ ${ }^{2}$ Department of Mathematics and Statistics, University of Helsinki, P.O. Box 68, \\ FI-00014 Helsinki, Finland \\ ${ }^{3}$ Department of Mathematics, Hunan University of Science and Technology, \\ Hunan, Xiangtan 411201, China \\ ${ }^{4}$ Department of Mathematics, Hangzhou Normal University, Zhejiang, \\ Hangzhou 310036, China
}

Correspondence should be addressed to Xuxin Yang, yangxx2002@sohu.com

Received 3 November 2011; Revised 10 December 2011; Accepted 22 January 2012

Academic Editor: F. Lobo Pereira

Copyright (c) 2012 Xuxin Yang et al. This is an open access article distributed under the Creative Commons Attribution License, which permits unrestricted use, distribution, and reproduction in any medium, provided the original work is properly cited.

We study the existence of solutions to the Duffing equation with impulses. By means of the Poincare-Birkhoff fixed point theorem under given conditions, we obtain the sufficient condition of existence of infinitely many solutions. Our results generalize those of T. R. Ding. An example is presented to demonstrate applications of our main result.

\section{Introduction}

Many evolution processes are characterized by the fact that at certain moments of time they experience abrupt changes in the value of the state variable. Theses processes are subject to short-time perturbations whose duration is negligible in comparison with the duration of the process. Consequently, it is natural to assume that these perturbations act instantaneously, that is, in the form of impulses. It is known, for example, that many biological phenomena involving thresholds, bursting rhythm models in medicine and biology, optimal control models in economics, pharmacokinetics, and frequency-modulated systems do exhibit impulsive effects. Thus impulsive differential equations, that is, differential equations involving impulse effects, appear as a natural description of observed evolution phenomena of several real world problems [1]. 
In its original form, the Duffing equation is a nonlinear second-order ODE which is used in physics to model oscillators. It is a well known example of a dynamical system exhibiting chaotic behavior. The equation is given by

$$
x^{\prime \prime}+\delta x^{\prime}+\alpha x+\beta x^{3}=\gamma \cos (\omega t)
$$

where the function $x=x(t)$ is the displacement at time $t$, and numbers $\delta, \alpha, \beta, \gamma$ and $\omega$ are constants.

In this paper, we study the following Duffing type model given by Ding in [2]

$$
x^{\prime \prime}+g(x)=f(x, t) \text {. }
$$

Clearly this equation contains the Duffing equation (1.1) without friction (i.e., $\delta=0$ ) as a special case. In [3], Ding considers (1.2) in the case where $g \in C(\mathbb{R}, \mathbb{R})$ is superlinear at infinity:

$$
\lim _{x \rightarrow \infty} \frac{g(x)}{x}=+\infty
$$

and the function $f \in C(\mathbb{R} \times \mathbb{R}, \mathbb{R})$ is $T$-periodic in $t$. By using the Poincare-Birkhoff fixed point theorem, Ding showed that there exist infinitely many periodic solutions to this equation.

By using a similar technique, Ming et al. [4] gave results concerning existence of infinitely many periodic solutions to the $p$-Laplace equation

$$
\left(\left|x^{\prime}\right|^{p-2} x^{\prime}\right)^{\prime}+g(x)=f(t, x), \quad p>1,
$$

where $g \in C(\mathbb{R}, \mathbb{R})$ is $p$-sublinear in the sense

$$
\lim _{|x| \rightarrow 0} \frac{g(x)}{|x|^{p-2} x}=+\infty
$$

and $f \in C(\mathbb{R} \times \mathbb{R}, \mathbb{R})$ is 1-periodic in $t$. For this problem, only partial results are known. For example, the conjecture is true if $g$ is even and superlinear at infinity and $f \equiv 0[5]$. For $f \neq 0$, more restrictions on $g$ are required $[6,7]$.

In this paper, by developing ideas of $[6,8,9]$, we extend this technique to the situation where $x$ is allowed to have impulses at given points. We consider the periodic solutions to the Duffing equation with impulses:

$$
\begin{gathered}
x^{\prime \prime}+g(x)=0, \quad t \neq t_{k}, \\
x\left(t_{k}^{+}\right)=a_{k} x\left(t_{k}\right), \quad k \in \mathbb{Z}, \\
x^{\prime}\left(t_{k}^{+}\right)=b_{k} x^{\prime}\left(t_{k}\right), \quad k \in \mathbb{Z},
\end{gathered}
$$

where $a_{k}>0, a_{k} b_{k}=1, a_{k+q}=a_{k}, t_{k+q}=t_{k}+T, 0<t_{1}<t_{2}<\cdots<t_{q}<T, g \in C(\mathbb{R}, \mathbb{R})$, and $\mathbb{Z}$ denotes the set of integers, $x\left(t_{k}^{+}\right)$and $x^{\prime}\left(t_{k}^{+}\right)$are right limits of $x(t)$ and $x^{\prime}(t)$ at $t=t_{k}$, 
respectively. Let PC $(\mathbb{R})=\left\{x: \mathbb{R} \rightarrow \mathbb{R} ; x(t)\right.$ is continuous everywhere except for $t_{k}$ at which $x\left(t_{k}^{+}\right)$and $x\left(t_{k}^{-}\right)$exist and $\left.x\left(t_{k}^{-}\right)=x\left(t_{k}\right), k \in \mathbb{Z}\right\} ; \mathrm{PC}^{1}(\mathbb{R})=\left\{x \in \mathrm{PC}(\mathbb{R}) ; x^{\prime}(t)\right.$ is continuous differentiable everywhere except for $t_{k}$ at which $x^{\prime}\left(t_{k}^{+}\right)$and $x^{\prime}\left(t_{k}^{-}\right)$exist and $x^{\prime}\left(t_{k}^{-}\right)=x^{\prime}\left(t_{k}\right), k \in$ $\mathbb{Z}\}$.

The paper is organized as follows. In Section 2, some preliminary results are given. In Section 3, we construct the twist map which is necessary in order to apply the fixedpoint results. Then, we obtain the main result for the existence of periodic solutions for the system by using the Poincaré-Birkhoff fixed-point theorem. In Section 4, an example is given to demonstrate the main result.

\section{Preliminaries}

The Poincare-Birkhoff fixed point theorem is a powerful tool in studying periodic solutions for the planar ODE's of the second order. There are several versions of this theorem, see $[8,10-13]$.

$\mathbb{R}^{2}$.

Let $(r, \theta)$ be a polar coordinates on $\mathbb{R}^{2}$ and $A=\left\{x \in \mathbb{R}^{2}: r_{1} \leq|x| \leq r_{2}\right\}$ an annulus on

Definition 2.1. A mapping $T$ is called as twist map if

$$
T:(r, \theta) \longrightarrow(h(r, \theta), \theta+l(r, \theta))
$$

where $h(r, \theta), l(r, \theta)$ are continuous on $A, 2 \pi$-periodic in $\theta$, and $l\left(r_{1}, \theta\right) l\left(r_{2}, \theta\right)<0$.

The proof of our main results is based on the following version of the Poincaré-Birkhoff theorem, due to Ding [2] (see also [10]).

Lemma 2.2 (see [2]). Let $R>r>0$. Suppose that $T: \mathbb{R}^{2} \rightarrow \mathbb{R}^{2}$ is an area preserving homeomorphism, such that $T$ is a twist map in an annulus

$$
A=\left\{x \in \mathbb{R}^{2}: r \leq|x| \leq R\right\}
$$

and $0 \in T(D)$, and $D=\{x:|x|<r\}$. Then $T$ has at least two fixed points in $A$.

For our convenience, we introduce the following condition:

$\left(H_{1}\right) g(x) x>0$ for $x \neq 0$ and

$$
\lim _{x \rightarrow 0} \frac{g(x)}{x}=+\infty
$$

Lemma 2.3. Assume that $g \in C(\mathbb{R}, \mathbb{R})$ and $\left(H_{1}\right)$ holds. Then for given $M>0$, there exists a constant $\delta=\delta(M)$ such that for any $\varepsilon>0$ there is a continuous function $g_{\varepsilon}$ such that

(1)

$$
\left|g_{\varepsilon}(x)-g(x)\right| \leq \varepsilon,
$$


(2) $g_{\varepsilon}$ has a form $g_{\varepsilon}(x)=M x$ near $x=0$, that is, there exists $\delta>0$ such that

$$
\frac{g_{\varepsilon}(x)}{x} \geq M \quad \text { for } 0<|\mathrm{x}| \leq \mathrm{ffi}
$$

and $g_{\varepsilon} \in C^{1}([-T, T] \backslash\{0\}, R)$, where $0<T<+\infty$.

(3) For a fixed $\lambda>0$,

$$
\lim _{x \rightarrow 0} \frac{G(\lambda x)}{G(x)}=\lambda^{2}
$$

where $G(x)=\int_{0}^{x} g_{\varepsilon}(s) d s$.

Proof. Choose $\rho>0$, and suppose $G(x) \geq v>0$ for $|x| \geq \rho$. Then by (2.3), there is a constant $\delta=\delta(M)$ such that

$$
\frac{g(x)}{x} \geq 2 M, \quad \text { for } 0<|x| \leq 2 \delta
$$

Choose $\mu>0$ such that $\mu \leq \varepsilon$, and let $\gamma>1$, such that $2 \gamma \mu^{2}+\mu \leq v^{2}$. Since $g(0)=0$, we can choose an interval $I_{0}=\left(-\delta_{0}, \delta_{0}\right)$ with $\delta_{0}<\delta$ such that

$$
|g(x)-M x| \leq \frac{\mu}{2}, \quad \forall x \in I_{0}
$$

For $x \neq 0$, we choose an interval $I_{x} \subset[-T, T]$ such that

$$
|g(y)-g(x)| \leq \frac{\mu}{2}, \quad \forall y \in I_{x}
$$

and $I_{x} \subset((1 / 2) x, 2 x)$ if $x>0, I_{x} \subset(2 x,(1 / 2) x)$ if $x<0$. We claim that there exists a covering of the interval $[-T, T]$, say $I_{0}, I_{i} \equiv I_{x_{i}}$ such that $I_{i}$ satisfies (2.9) for all $i=0,1,2, \ldots, n$. Here we only consider the case of $\left[-T,-\delta_{0}\right]\left(\delta_{0}<T\right)$. Because $g$ is uniformly continuous on the closed interval $\left[-T,-\delta_{0}\right]$, there exists a $\sigma>0$ independent of $x, y \in\left[-T,-\delta_{0}\right]$ such that $|g(y)-g(x)| \leq$ $\mu / 2$ for $|x-y| \leq \sigma$. Define a sequence $\sigma_{n}(n \geq 1)$ by

$$
\sigma_{n}=\sup \left\{\sigma:\left|g(y)-g\left(-T+\sum_{i=1}^{n-1} \sigma_{i}\right)\right| \leq \frac{1}{2} \mu, \quad \forall y \in\left[-T+\sum_{i=1}^{n-1} \sigma_{i},-T+\sigma+\sum_{i=1}^{n-1} \sigma_{i}\right]\right\}
$$

and $\sigma_{0}=0$. It is easy to check that $\sigma_{n}>0, n=1,2, \ldots$ Now we assume that $\sum_{i=1}^{\infty} \sigma_{i} \leq T-\delta_{0}$. Since $g$ is continuous on the closed interval $[-T, T]$, we obtain that

$$
\left|g\left(-T+\sum_{i=1}^{N-1} \sigma_{i}+\frac{1}{N+1}\right)-g\left(-T+\sum_{i=1}^{N-1} \sigma_{i}\right)\right| \leq \frac{1}{2} \mu \text {, }
$$


where $N$ is a sufficiently large positive integer, which implies $\sigma_{N} \geq(N+1)^{-1}$ for sufficiently large positive integer $N$. On the other hands, $\sum_{k=N}^{\infty} k^{-1}=\infty$, which implies $\sum_{i=1}^{\infty} \sigma_{i}=\infty$, a contradiction. We also assume that $I_{i} \cap I_{j}=\emptyset$ for $|i-j|>1$.

Let $\phi_{0}, \phi_{1}, \phi_{2}, \ldots, \phi_{n}$ be resolution of unit that corresponds to $I_{i}(0 \leq i \leq n)$ such that $\sum_{i=0}^{n} \phi_{i}(x)=1$, for $x \in\left(x_{i}, x_{i+1}\right)$, and supp $\phi_{i} \subset I_{i}$ for each $i=0,1, \ldots, n$. Then, for $x \in[-T, T]$, we define

$$
g_{\varepsilon}(x)=\phi_{0}(x) M x+\sum_{i=1}^{n} \phi_{i}(x) g\left(x_{i}\right)
$$

By (2.9) and (2.12), we have

$$
\left|g_{\varepsilon}(x)-g(x)\right| \leq \phi_{0}(x)|M x-g(x)|+\sum_{i=1}^{n} \phi_{i}(x)\left|g(x)-g\left(x_{i}\right)\right| \leq \mu \leq \varepsilon,
$$

proving the claim (2.4). Furthermore

$$
\left|g_{\varepsilon}(x)-g(x)\right| \leq \mu
$$

To see (2.5), we suppose that $0<|x|<\delta$ and that, for $i \geq 1$, we have $\phi_{i}(x) \neq 0$ and $1 / 2 \leq$ $x_{i} / x \leq 2$. It follows that $\left|x_{i}\right| \leq 2|x| \leq 2 \delta$ and, by (2.7),

$$
\frac{g\left(x_{i}\right)}{x_{i}} \geq 2 M
$$

Then, by the above inequality and (2.12), we have

$$
\frac{g_{\varepsilon}(x)}{x}=\phi_{0}(x) M+\sum_{i=1}^{n} \phi_{i}(x) \frac{g\left(x_{i}\right) x_{i}}{x_{i} x} \geq \phi_{0}(x) M+\sum_{i=1}^{n} \phi_{i}(x) 2 \frac{M}{2}=M .
$$

Since $g_{\varepsilon}$ has the form $g_{\varepsilon}(x)=M x$ near $x=0, G(x)$ has the form $G(x)=M x^{2} / 2$ near $x=0$, which implies (2.6).

Let $g \in C(\mathbb{R}, \mathbb{R})$ and satisfies the condition $\left(H_{1}\right)$, and let $g_{\varepsilon}$ be as Lemma 2.3. Consider the initial value problem for the following system:

$$
\begin{gathered}
x^{\prime}=-y, \\
y^{\prime}=g_{\varepsilon}(x), \quad t \neq t_{k}, \\
x\left(t_{k}^{+}\right)=a_{k} x\left(t_{k}\right), \\
y\left(t_{k}^{+}\right)=b_{k} y\left(t_{k}\right), \quad k=1,2, \ldots, \\
x(0)=x_{0}, \quad y(0)=y_{0} .
\end{gathered}
$$


Lemma 2.4. For any sufficiently small $\left(x_{0}, y_{0}\right)$ the system (2.17) has a unique solution pair $x(t)=$ $x\left(t, x_{0}, y_{0}\right), y(t)=y\left(t, x_{0}, y_{0}\right)$. Moreover, the functions $x, y$ continuously depend on $x_{0}, y_{0}$.

Proof. Obviously, we only need to prove that the conclusion holds for the system without impulses

$$
\begin{gathered}
x^{\prime}=-y, \\
y^{\prime}=g_{\varepsilon}(x), \\
x(0)=x_{0}, \quad y(0)=y_{0} .
\end{gathered}
$$

Since $g_{\varepsilon}$ is continuous, the initial value problem (2.18) has a solution by the Peano's method.

Next, we show that the solution of (2.17) is unique. For $\gamma>0$ is sufficiently small, the initial value problem:

$$
\begin{gathered}
x^{\prime}=-y, \\
y^{\prime}=g_{\varepsilon}(x), \quad t \geq \gamma, \\
x(\gamma)=\alpha, \quad y(\gamma)=\beta,
\end{gathered}
$$

where $\alpha \in \mathbb{R}, \beta \in \mathbb{R}$, has a unique solution since $g_{\varepsilon} \in C^{1}([\gamma, d], \mathbb{R})$. Hence we need only to show that the solution of (2.18) is unique for $0 \leq t \leq \gamma$. If $x_{0}=0$, (2.18) reduces to

$$
\begin{gathered}
x^{\prime \prime}+M x=0, \\
x(0)=0, \quad x^{\prime}(0)=-y_{0},
\end{gathered}
$$

because $g_{\varepsilon}$ has the form $g_{\varepsilon}(x)=M x$ near $x=0$, and thus our claim holds. If $x_{0} \neq 0, g_{\varepsilon} \in$ $C^{1}\left(\Omega\left(x_{0}\right), \mathbb{R}\right)$, where $\Omega\left(x_{0}\right)=\left\{x:\left|x-x_{0}\right| \leq \gamma\right\}$. So the solution of (2.18) is unique for $0 \leq t \leq \gamma$. Continuous dependence on the initial data follows from the uniqueness.

\section{Main Result}

Our main result is the following theorem. This result generalizes Theorem 2.1 of Ding [3].

Theorem 3.1. Under the assumption (2.3), the Duffing equation (1.6) has an infinite sequence of solutions $\left\{x_{n}\right\}$ and $\left\|x_{n}\right\|_{\mathrm{PC}^{1}[0, \mathrm{~T}]} \rightarrow 0$, as $n \rightarrow \infty$, where

$$
\left\|x_{n}\right\|_{\mathrm{PC}^{1}[0, T]}=\max \left\{\sup _{t \in[0, T]}\left\{\left|x_{n}(t)\right|\right\}, \sup _{t \in[0, T]}\left\{\left|x_{n}^{\prime}(t)\right|\right\}\right\} .
$$

In order to prove the main result, we need the following lemmas. First we construct the twist map. Next we consider the system (2.17), our goal is to control the behavior of the norms $r(t)=\sqrt{x(t)^{2}+y(t)^{2}}$ at the points $p(t)=(x(t), y(t)) \in \mathbb{R}^{2}$. 
Lemma 3.2. Suppose that $p(t)=(x(t), y(t))$, where $t \in[0, T]$ is a solution to the system (2.17). There exists a constant $R_{0}>0$ and functions $d_{1}, d_{2}, \varepsilon:\left(0, R_{0}\right] \rightarrow \mathbb{R}_{+}$such that if $\varepsilon \leq \varepsilon(R)$, and $r(0)=R<R_{0}$, then
(1) $d_{1}(R) \leq r(t) \leq d_{2}(R)$,
(2) $\lim _{R \rightarrow 0} d_{1}(R)=\lim _{R \rightarrow 0} d_{2}(R)=0$.

Proof. Define the Liapunov function

$$
F(x, y)=\frac{1}{2} y^{2}+\int_{0}^{x} g_{\varepsilon}(s) d s
$$

We compute the differential of $F$ along the curve $p(t)=(x(t), y(t))$, where $t \in[0, T]$ :

$$
\frac{d F(p(t))}{d t}=y y^{\prime}+g_{\varepsilon}(x) x^{\prime}=y g_{\varepsilon}(x)+g_{\varepsilon}(x)(-y)=0,
$$

for $t \neq t_{k}$.

From Lemma 2.3, we see that for a fixed $\lambda>0$, there exist $c_{1}(\lambda) \geq 0, c_{2}(\lambda) \geq 0$ such that

$$
c_{2}(\lambda) G(x) \leq G(\lambda x) \leq c_{1}(\lambda) G(x),
$$

where $G(x)=\int_{0}^{x} g_{\varepsilon}(s) d s$, when $|x|$ is sufficiently small. We have

$$
F\left(p\left(t_{k}^{+}\right)\right)=\frac{1}{2} y\left(t_{k}^{+}\right)^{2}+\int_{0}^{x\left(t_{k}^{+}\right)} g_{\varepsilon}(s) \mathrm{d} s=\frac{1}{2} b_{k}^{2} y\left(t_{k}\right)^{2}+\int_{0}^{a_{k} x\left(t_{k}\right)} g_{\varepsilon}(s) d s
$$

From (3.4), we have

$$
\begin{aligned}
\min \left\{b_{k}^{2}, c_{2}\left(a_{k}\right)\right\} F\left(p\left(t_{k}\right)\right) \leq F\left(p\left(t_{k}^{+}\right)\right) & \leq \max \left\{b_{k^{\prime}}^{2}, c_{1}\left(a_{k}\right)\right\}\left(\frac{1}{2} y\left(t_{k}\right)^{2}+\int_{0}^{x\left(t_{k}\right)} g_{\varepsilon}(s) d s\right) \\
& \leq \max \left\{b_{k}^{2}, c_{1}\left(a_{k}\right)\right\} F\left(p\left(t_{k}\right)\right) .
\end{aligned}
$$

By using an impulsive integer inequality, we obtain

$$
F(p(0)) \prod_{0<t_{k}<t} \min \left\{b_{k}^{2}, c_{2}\left(a_{k}\right)\right\} \leq F\left(p\left(t_{k}\right)\right) \leq F(p(0)) \prod_{0<t_{k}<t} \max \left\{b_{k^{\prime}}^{2}, c_{1}\left(a_{k}\right)\right\}
$$

It follows that

$$
B_{1} F(p(0)) \leq F(p(t)) \leq A_{1} F(p(0)), \quad \forall t \in[0, T],
$$


where $A_{1}=\prod_{0<t_{k}<1} \max \left\{b_{k^{\prime}}^{2}, c_{1}\left(a_{k}\right)\right\}$, and $B_{1}=\prod_{0<t_{k}<1} \min \left\{b_{k^{\prime}}^{2} c_{2}\left(a_{k}\right)\right\}$. We also have

$$
B_{1} \min _{|p|=R} F(p) \leq F(p(t)) \leq A_{1} \max _{|p|=R} F(p), \quad \forall t \in[0, T]
$$

and thus there exist positive constants $d_{1}(R), d_{2}(R)$ such that

$$
d_{1}(R) \leq r(t) \leq d_{2}(R)
$$

Furthermore, we have

$$
\lim _{R \rightarrow 0} d_{1}(R)=\lim _{R \rightarrow 0} d_{2}(R)=0 .
$$

Next, we consider the following system:

$$
\begin{gathered}
x^{\prime}=-y, \\
y^{\prime}=g(x), \quad t \neq t_{k}, \\
x\left(t_{k}^{+}\right)=a_{k} x\left(t_{k}\right), \\
y\left(t_{k}^{+}\right)=b_{k} y\left(t_{k}\right), \quad k=1,2, \text { and so forth. }
\end{gathered}
$$

It follows from Lemma 3.2 that we can obtain a solution of (3.12) which can be extended to the interval $[0, T]$ if $\left|p_{0}\right|=\left|\left(x_{0}, y_{0}\right)\right| \leq R_{0}$. Now we can define the Poincaré map $\phi$ :

$$
\left(x_{0}, y_{0}\right) \longmapsto\left(x\left(T, x_{0}, y_{0}\right), y\left(T, x_{0}, y_{0}\right)\right)=\left(x_{T}, y_{T}\right)
$$

where $\left(x\left(t, x_{0}, y_{0}\right), y\left(t, x_{0}, y_{0}\right)\right.$ is the solution of (3.12) corresponding to the initial data $\left(x_{0}, y_{0}\right)$. Denote by $r(t), \theta(t)$ the norm and the polar angle of $p(t)=(x(t), y(t)) \in \mathbb{R}^{2}, r(t)=$ $\sqrt{x(t)^{2}+y(t)^{2}}$, respectively. Then

$$
\begin{gathered}
\cos \theta(t)=\frac{x(t)}{\sqrt{x^{2}(t)+y^{2}(t)}}, \quad \sin \theta(t)=\frac{y(t)}{\sqrt{x^{2}(t)+y^{2}(t)}}, \quad t \neq t_{k}, \\
\cos \theta\left(t_{k}^{+}\right)=\frac{x\left(t_{k}^{+}\right)}{\sqrt{x\left(t_{k}^{+}\right)^{2}+y\left(t_{k}^{+}\right)^{2}}}=\frac{a_{k} x\left(t_{k}\right)}{\sqrt{a_{k}^{2} x\left(t_{k}\right)^{2}+b_{k}^{2} y\left(t_{k}\right)^{2}}}, \\
\sin \theta\left(t_{k}^{+}\right)=\frac{y\left(t_{k}^{+}\right)}{\sqrt{x\left(t_{k}^{+}\right)^{2}+y\left(t_{k}^{+}\right)^{2}}}=\frac{b_{k} y\left(t_{k}\right)}{\sqrt{a_{k}^{2} x\left(t_{k}\right)^{2}+b_{k}^{2} y\left(t_{k}\right)^{2}}}, \quad k=1,2, \ldots
\end{gathered}
$$


Write $\left(r_{0}, \theta_{0}\right)=(r(0), \theta(0))$ and $\left(r_{T}, \theta_{T}\right)=(r(T), \theta(T))$. Then, the map $\phi$ can be expressed in the polar coordinates as

$$
r_{T}=h\left(r_{0}, \theta_{0}\right), \quad \theta_{T}=\theta_{0}+l\left(r_{0}, \theta_{0}\right),
$$

where $h$ and $l$ are continuous and $2 \pi$-periodic in $\theta_{0}$. In order to apply the Poincaré-Birkhoff fixed point theorem, we need to estimate the difference $\theta_{T}-\theta_{0}$.

Lemma 3.3. For $R>0$ there is a constant $K(R)$, and $r_{0}=R$, then $\theta_{T}-\theta_{0}=l\left(R, \theta_{0}\right) \leq K(R)$.

Proof. We estimate the differential of the polar angle $\theta(t)$ along the solution curve $(x(t), y(t))$ as follows:

$$
\frac{d \theta(t)}{d t}=\frac{x y^{\prime}-y x^{\prime}}{x^{2}+y^{2}}=\frac{x g_{\varepsilon}(x)+y^{2}}{x^{2}+y^{2}}, \quad t \neq t_{k}
$$

For $t=t_{k}$, we have

$$
\tan \theta\left(t_{k}^{+}\right)=\frac{y\left(t_{k}^{+}\right)}{x\left(t_{k}^{+}\right)}=\frac{b_{k}}{a_{k}} \tan \theta\left(t_{k}\right)
$$

From (3.17), we obtain

$$
\frac{\theta\left(t_{k}^{+}\right)}{\theta\left(t_{k}\right)}=\frac{\arctan \left(\left(b_{k} / a_{k}\right) \tan \theta\left(t_{k}\right)\right)}{\theta\left(t_{k}\right)}
$$

Next we construct the function, for $a>0$, by

$$
h(x)= \begin{cases}\frac{1}{x} \arctan (a \tan x), & \text { if } x \neq 0 \\ a, & \text { if } x=0\end{cases}
$$

Then, it is easy to see there exist $\alpha(a)$ and $\beta(a)$ such that

$$
0<\alpha(a) \leq h(x) \leq \beta(a)<\infty
$$

From (3.18) and (3.20), we have

$$
0<\alpha\left(\frac{b_{k}}{a_{k}}\right) \theta\left(t_{k}\right) \leq \theta\left(t_{k}^{+}\right) \leq \beta\left(\frac{b_{k}}{a_{k}}\right) \theta\left(t_{k}\right)<\infty .
$$

Furthermore, by Lemma 3.2, we have $0<d_{1}(R) \leq \sqrt{x^{2}+y^{2}} \leq d_{2}(R)$, and (3.16), hence

$$
\frac{d \theta(t)}{d t} \leq K(R)
$$


From (3.21) and (3.22), we obtain

$$
\theta(T) \leq \theta_{0} \prod_{0<t_{k}<T} \beta\left(\frac{b_{k}}{a_{k}}\right)+\int_{0}^{T} \prod_{s<t_{k}<T} \beta\left(\frac{b_{k}}{a_{k}}\right) K(R) d s .
$$

This implies that there exists an integer $m=m(R)$ such that

$$
\theta_{T}-\theta_{0}+2 m \pi=l\left(R, \theta_{0}\right)+2 m \pi<0, \quad \forall \theta_{0} \in[0,2 \pi]
$$

Lemma 3.4. There is $r<R$ such that

$$
\theta_{T}-\theta_{0}+2 m \pi=l\left(r, \theta_{0}\right)+2 m \pi>0, \quad \forall \theta_{0} \in[0,2 \pi] .
$$

So the map $\phi$ is the twist map in the annular region $A=\left\{(x, y): r \leq \sqrt{x^{2}+y^{2}} \leq R\right\}$.

Proof. Let $M$ be a large number to be chosen later. Let $g$ be the approximation function defined in Lemma 2.3. In particular we have

$$
\frac{g_{\varepsilon}(x)}{x} \geq M, \quad \text { if } 0<|x| \leq \delta .
$$

Choose $r$ such that $d_{2}(r)<\delta$. If the initial data $r_{0}=\sqrt{x^{2}+y^{2}}=r$, then by Lemma 3.2 the solution $(x, y)=(x(t), y(t))$ satisfies $\sqrt{x^{2}(t)+y^{2}(t)} \leq d_{2}(r)<\delta$, and hence $g_{\varepsilon}(x) \geq M x$.

To estimate the difference $\theta_{T}-\theta_{0}$, it is convenient to introduce another angle variable $\Theta$, defined by the formulas:

$$
\cos \Theta(t)=\frac{M^{1 / 2} x(t)}{\sqrt{M x^{2}(t)+y^{2}(t)}}, \quad \sin \Theta(t)=\frac{y(t)}{\sqrt{M x^{2}(t)+y^{2}(t)}}, \quad t \neq t_{k} .
$$

It follows that

$$
\begin{gathered}
\tan \Theta\left(t_{k}\right)=\frac{y\left(t_{k}\right)}{M^{1 / 2} x\left(t_{k}\right)}, \quad t=t_{k} \\
\Theta\left(t_{k}^{+}\right)=\arctan \left(\frac{y\left(t_{k}^{+}\right)}{M^{1 / 2} x\left(t_{k}^{+}\right)}\right)=\arctan \left(\frac{b_{k} y\left(t_{k}\right)}{M^{1 / 2} a_{k} x\left(t_{k}\right)}\right)=\arctan \left(\frac{b_{k}}{a_{k}} \tan \Theta\left(t_{k}\right)\right), \quad t=t_{k} .
\end{gathered}
$$


Note that by (3.27) and (3.14) $\cos \Theta$ and $\cos \theta$, respectively, $\sin \Theta$ and $\sin \theta$, have the same sign. Thus the two angles $\Theta$ and $\theta$ always lie in the same quarter and $\Theta=\theta+\alpha+2 k \pi, k \in$ $\mathbb{Z},-\pi / 2 \leq \alpha \leq \pi / 2$, if and only if the polar angle $\theta$ is in the same interval. Hence, if $|\theta-\Theta|<$ $\pi / 2$ at the initial time $t=0$, this estimate will remain true along a continuous curve not passing through the origin.

We have

$$
\frac{d \Theta(t)}{d t}=\frac{1}{1+\left(y / M^{1 / 2} x\right)^{2}} \times \frac{y^{\prime} M^{1 / 2} x-y M^{(1 / 2) x^{\prime}}}{M x^{2}}=\frac{M^{1 / 2}\left(x g_{\varepsilon}(x)+y^{2}\right)}{M x^{2}+y^{2}} \geq M^{1 / 2}
$$

By (3.20), (3.28), and (3.29) we have

$$
\begin{gathered}
\Theta^{\prime}\left(t_{k}\right) \geq M^{1 / 2}, \\
\Theta\left(t_{k}^{+}\right) \geq \alpha\left(\frac{b_{k}}{a_{k}}\right) \Theta\left(t_{k}\right),
\end{gathered}
$$

and by (3.30), it follows that

$$
\Theta(T) \geq \Theta(0) \prod_{0<t_{k}<T} \alpha\left(\frac{b_{k}}{a_{k}}\right)+\int_{0}^{T} \prod_{s<t_{k}<T} \alpha\left(\frac{b_{k}}{a_{k}}\right) M^{1 / 2} d s
$$

So there exist $\sigma_{1} \in \mathbb{R}$ and $\sigma_{2}>0$, such that

$$
\Theta(T) \geq \sigma_{1}+\sigma_{2} M^{1 / 2}
$$

Recall the relations $\Theta=\theta+\alpha+2 k \pi, k \in \mathbb{Z},-\pi / 2 \leq \alpha \leq \pi / 2$. Then, by (3.32), we have

$$
\begin{aligned}
\theta(T)-\theta(0)+2 m \pi & =\Theta(T)-\alpha-2 k \pi-\theta(0)+2 m \pi \\
& \geq \sigma_{1}+\sigma_{2} M^{1 / 2}-\alpha-2 k \pi-\theta(0)+2 m \pi>0, \quad \forall \theta_{0} \in[0,2 \pi]
\end{aligned}
$$

We choose an integer $m=m(r)$ such that

$$
\theta_{T}-\theta_{0}+2 m \pi=l\left(r, \theta_{0}\right)+2 m \pi>0, \quad \forall \theta_{0} \in[0,2 \pi]
$$

provided that $M$ is large enough. 
Proof of Theorem 3.1. Let $\phi$ be the map defined (3.13), or equivalently by (3.15). Define the mappings:

$$
\begin{gathered}
\Phi_{0}:\left(x_{0}, y_{0}\right) \longrightarrow\left(x\left(t_{1}, x_{0}, y_{0}\right), y\left(t_{1}, x_{0}, y_{0}\right)\right)=\left(x_{1}, y_{1}\right), \\
\Phi_{0}^{*}:\left(x_{1}, y_{1}\right) \longrightarrow\left(a_{1} x\left(t_{1}, x_{0}, y_{0}\right), b_{1} y\left(t_{1}, x_{0}, y_{0}\right)\right)=\left(x_{1}^{*}, y_{1}^{*}\right), \\
\Phi_{i}:\left(x_{i}^{*}, y_{i}^{*}\right) \longrightarrow\left(x\left(t_{i+1}, x_{0}, y_{0}\right), y\left(t_{i+1}, x_{0}, y_{0}\right)\right)=\left(x_{i+1}, y_{i+1}\right), \\
\Phi_{i}^{*}:\left(x_{i+1}, y_{i+1}\right) \longrightarrow\left(a_{i+1} x_{i+1}, b_{i+1} y_{i+1}\right)=\left(x_{i+1}^{*}, y_{i+1}^{*}\right), \quad i=1, \ldots, q-1, \\
\Phi_{q}:\left(x_{q}^{*}, y_{q}^{*}\right) \longrightarrow\left(x\left(T, x_{0}, y_{0}\right), y\left(T, x_{0}, y_{0}\right)\right) .
\end{gathered}
$$

When $a_{k} b_{k}=1, \Phi_{i}(0 \leq i \leq q), \Phi_{j}^{*}(0 \leq j \leq q-1)$ are area-preserving mappings. Since

$$
\phi=\Phi_{q} \circ \Phi_{q-1}^{*} \circ \Phi_{q-1} \circ \cdots \circ \Phi_{0}^{*} \circ \Phi_{0}
$$

$\phi$ is an area-preserving mapping. Obviously, $\phi(0,0)=(0,0) \in D=\left\{(x, y): x^{2}+y^{2}<r^{2}\right\}$. Lemmas 3.3, 3.4 imply that $\phi$ is a twist map on the annulus $A=\left\{(x, y): r^{2} \leq x^{2}+y^{2} \leq R^{2}\right\}$ for sufficiently small $\varepsilon$. Now it follows from the result of Ding, Lemma 2.2 given in the introduction, that $\phi$ has at least two fixed points in $A$. Let $\left(x_{\varepsilon_{i}}(t), y_{\varepsilon_{i}}(t)\right)$ be one of the corresponding periodic solutions of (3.12). By Lemma 3.2, we have

$$
d_{1}(r) \leq \sqrt{x_{\varepsilon_{i}}^{2}(t)+y_{\varepsilon_{i}}^{2}(t)} \leq d_{2}(R)
$$

By the Arzela-Ascoli theorem, a sequence of $\left\{x_{\varepsilon_{i}}(t), y_{\varepsilon_{i}}(t)\right\}$ converges to $(x(t), y(t))$ as $\varepsilon_{i} \rightarrow 0$. Then, $(x(t), y(t))$ satisfies (3.12), and $(x(t), y(t))$ is a periodic solution for the system (1.6) with $d_{1}(r) \leq \sqrt{x^{2}(t)+y^{2}(t)} \leq d_{2}(R)$. Since $R$ is arbitrary, we obtain an infinite sequence of periodic solutions for system (1.6) with small amplitudes.

\section{Discussion}

To the best of our knowledge, there are few papers concerned with the existence of periodic solutions for impulsive equations by means of the Poincaré-Birkhoff fixed point theorem. The goal of this paper is to fill the gap in this area. Our main result expands the applied scope of the work of Ding [3] by allowing the equation to have impulses. Application of the result obtained is illustrated by Example 4.1 given below in which the conclusion does not follow from earlier result, as it does not allow impulses.

Example 4.1. Consider the equation

$$
\begin{gathered}
x^{\prime \prime}(t)+\sqrt[3]{x(t)}=0, \quad t \neq t_{k}, \\
x\left(t_{k}^{+}\right)=a_{k} x\left(t_{k}\right), \quad x^{\prime}\left(t_{k}^{+}\right)=b_{k} x^{\prime}\left(t_{k}\right), \quad k \in \mathbb{Z},
\end{gathered}
$$

where $a_{k}>0, a_{k} b_{k}=1, a_{k+q}=a_{k}, t_{k+q}=t_{k}+T, 0<t_{1}<t_{2}<\cdots<t_{q}<T$. 
In fact, in (4.1), $g(x)=\sqrt[3]{x}$. Then, $x g(x) \geq 0$ and $\lim _{x \rightarrow 0} x^{-1} g(x)=+\infty$. The condition $\left(H_{1}\right)$ is satisfied and by Theorem 3.1, (4.1) has an infinite sequence of periodic solutions.

In addition, this paper is the new application for the work of Ding [10] in the impulsive differential equations.

\section{Acknowledgments}

This work is supported by the NNSF of China (10871062), Scientific Research Fund of Hunan Provincial Education Department (10B017), and Hunan Provincial Natural Science Foundation of China (09JJ3010).

\section{References}

[1] V. Lakshmikantham, D. D. Bănov, and P. S. Simeonov, Theory of Impulsive Differential Equations, vol. 6 of Series in Modern Applied Mathematics, World Scientific Publishing, Teaneck, NJ, USA, 1989.

[2] W. Y. Ding, "Fixed points of twist mappings and periodic solutions of ordinary differential equations," Acta Mathematica Sinica, vol. 25, no. 2, pp. 227-235, 1982.

[3] T. R. Ding, "An infinite class of periodic solutions of periodically perturbed Duffing equations at resonance," Proceedings of the American Mathematical Society, vol. 86, no. 1, pp. 47-54, 1982.

[4] X. Ming, S. Wu, and J. Liu, "Periodic solutions for the 1-dimensional p-equation," Journal of Mathematical Analysis and Applications, vol. 325, no. 2, pp. 879-888, 2007.

[5] A. Ambrosetti and P. H. Rabinowitz, "Dual variational methods in critical point theory and applications," Journal of Functional Analysis, vol. 14, pp. 349-381, 1973.

[6] W. Ding, J. Mi, and M. Han, "Periodic boundary value problems for the first order impulsive functional differential equations," Applied Mathematics and Computation, vol. 165, no. 2, pp. 433-446, 2005.

[7] M. Struwe, "Infinitely many critical points for functionals which are not even and applications to superlinear boundary value problems," Manuscripta Mathematica, vol. 32, no. 3-4, pp. 335-364, 1980.

[8] M. Y. Jiang, "A Landersman-Lazer type theorem for periodic solutions of resonant asymmeetric pLaplace equation," Research report 101, School of Mathematics Science, Peking University, 2002.

[9] B. Liu, "Multiplicity results for periodic solutions of a second order quasilinear ODE with asymmetric nonlinearities," Nonlinear Analysis. Theory, Methods E Applications, vol. 33, no. 2, pp. 139-160, 1998.

[10] W. Y. Ding, "A generalization of the Poincaré-Birkhoff theorem," Proceedings of the American Mathematical Society, vol. 88, no. 2, pp. 341-346, 1983.

[11] J. Franks, "Generalizations of the Poincaré-Birkhoff theorem," Annals of Mathematics Second Series, vol. 128, no. 1, pp. 139-151, 1988.

[12] H. Jacobowitz, "Periodic solutions of $x^{\prime \prime}+f(t, x)=0$ via the Poincaré-Birkhoff theorem," Journal of Differential Equations, vol. 20, no. 1, pp. 37-52, 1976.

[13] C. Rebelo and F. Zanolin, "Twist conditions and periodic solutions of differential equations," in Proceedings of Dynamic Systems and Applications, vol. 2, pp. 469-476, Dynamic, Atlanta, Ga, USA, 1996. 


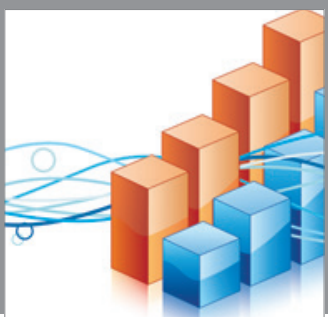

Advances in

Operations Research

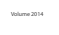

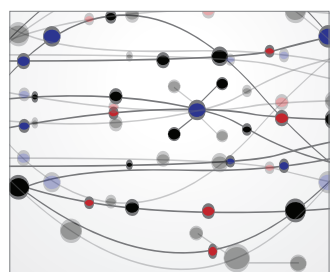

\section{The Scientific} World Journal
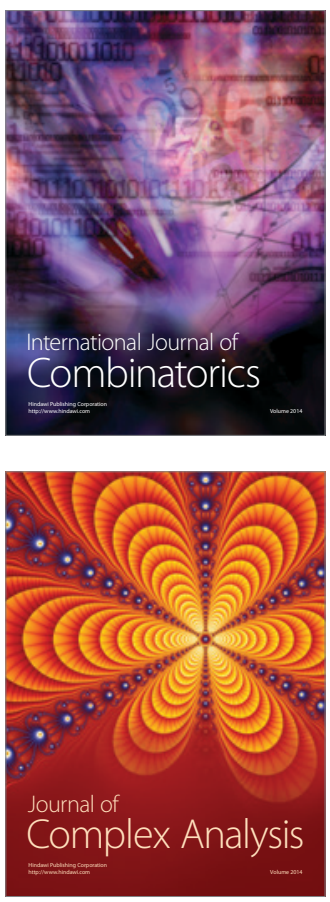

International Journal of

Mathematics and

Mathematical

Sciences
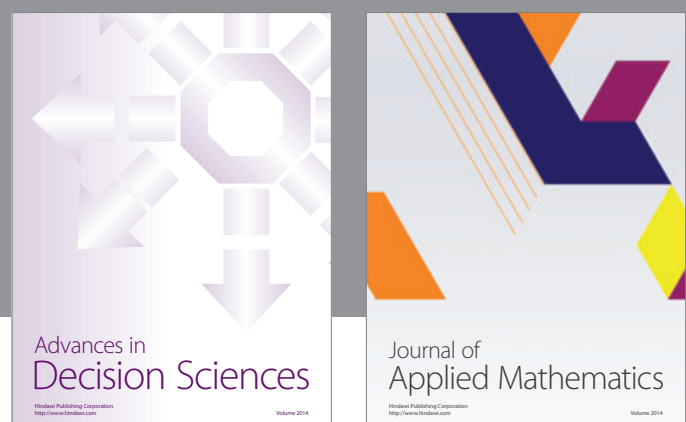

Journal of

Applied Mathematics
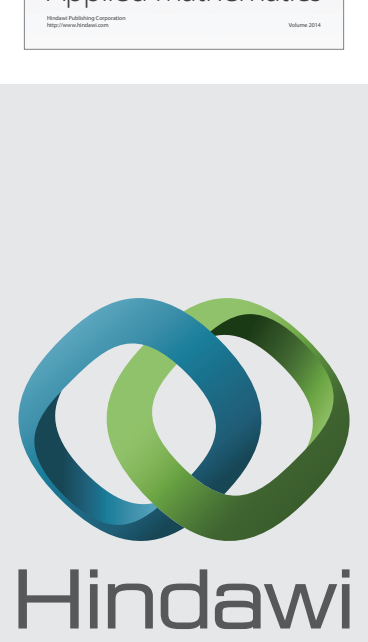

Submit your manuscripts at http://www.hindawi.com
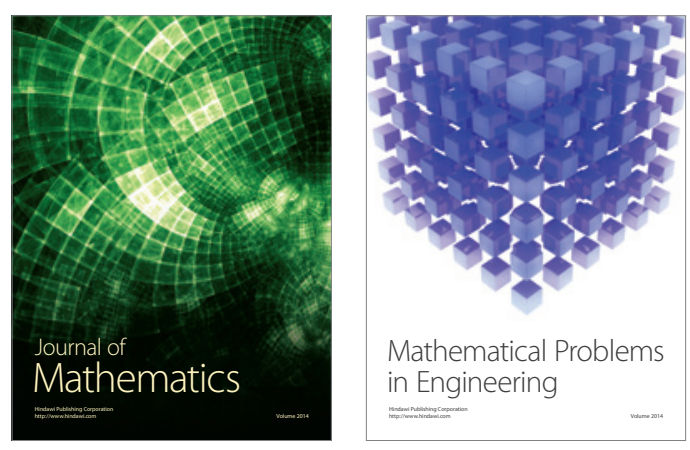

Mathematical Problems in Engineering
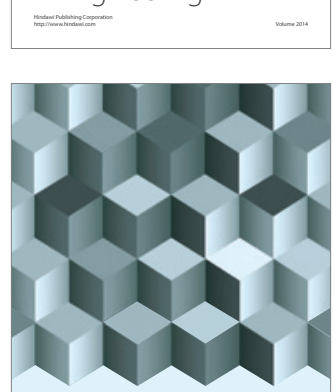

Journal of

Function Spaces
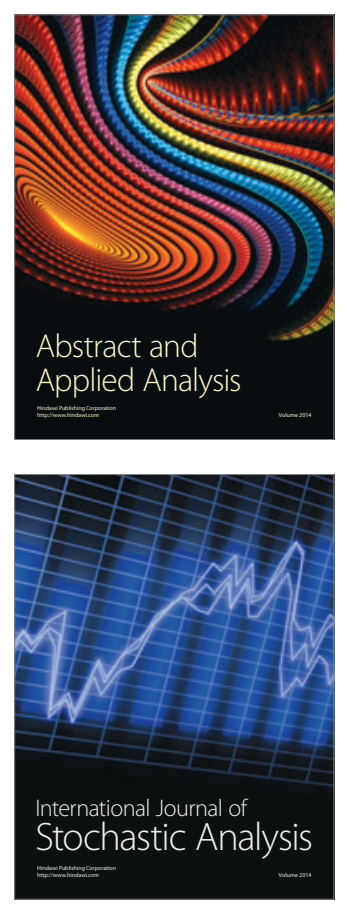

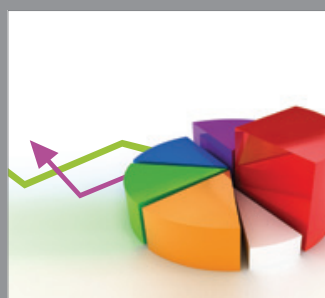

ournal of

Probability and Statistics

Promensencen
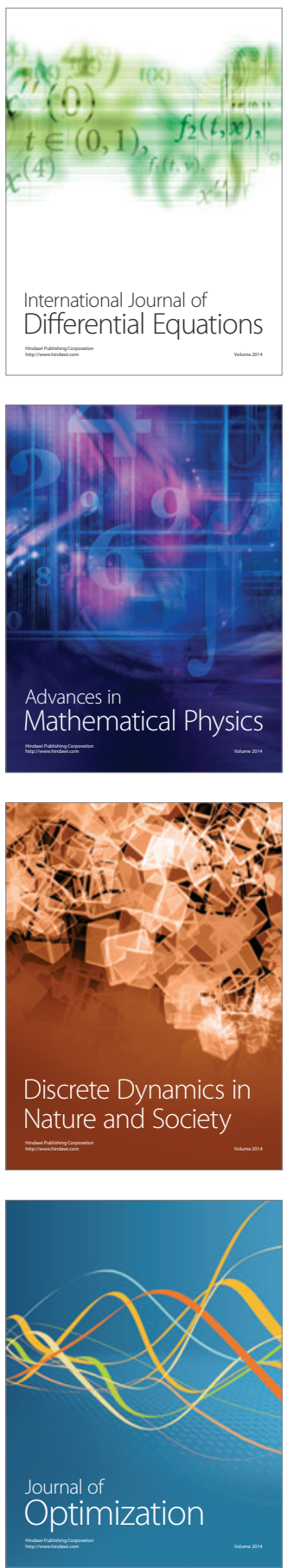